\title{
The simulation and experiment on mechanical properties of materials based on dispersed characters in micro-level
}

\author{
Yuefeng $\mathrm{Li}^{1, \mathrm{a}}$, Haoyang Wang ${ }^{1}$, Guanglin Wang ${ }^{1}$, Li Sun ${ }^{2}$ \\ ${ }^{1}$ School of Mechanical Engineering, Harbin Institute of Technology, Harbin, China, 150001 \\ ${ }^{2}$ School of Electrical Engineering \& Automation, Harbin Institute of Technology, Harbin, China, \\ 150001 \\ aemail:lilu1001@163.com
}

Keywords: Dispersed characters; Finite element analysis; Experimental verification

\begin{abstract}
To solve the defect of common finite element analysis which cannot imitate the micro-structure and mechanical properties of materials, a new kind of FEA model is founded to simulate the metallic micro-structure based on the dispersed mechanical characters. Some research is performed by this model on the low-cycle fatigue process where top process in the cycle is slightly under the yield stress. Firstly a theory model towards the process of elastic and plastic deformation coupled with damage accumulation is founded. Secondly some simulation is performed by the new FEA model. Finally the simulation is verified by tensile and compressing experiment. The experimental results show that the new FEA model can simulate the deformation of metallic materials in micro-level accurately.
\end{abstract}

\section{Introduction}

With the development of the theory of finite element analysis, the technique to solve the analysis of production structure, the calculation of stress distribution and the simulation of fatigue process by use of FEA software is widely used[1-2]. In the FEA software Ansys and Abaqus, the module to research the fatigue process is integrated. By this module, the remaining life of the mechanical components under cycled load can be obtained. However, for some machine which has a small size, its fatigue and crack during the use in a long time is related with the distribution of grain and defect in the micro-level[3]. With the common FEA model where the mechanical properties in the machine is distributed evenly the fatigue failure life and the location of the initial crack cannot be predicted. In this paper a new kind of FEA model is founded to simulate the metallic micro-structure based on the dispersed mechanical characters. Some simulation is performed on the process of low-cycled fatigue failure by this model. At last a series of tensile tests are designed and performed in the hydraulic fatigue testing machine to verify the results of FEA simulation.

\section{Theory background}

For common machine there are both elastic and plastic deformation in the process of fatigue failure. There are also many process of loading and unloading at the same time. According to the theory of metallic elastic and plastic deformation, the incremental stress-strain model is proper to express this process. In the process of damage accumulation, the nonlinear fatigue damage accumulation law without damage caused from elastic deformation is used. At the same time, a whole constitutive model for fatigue failure is founded by performing a coupling calculation between elastic and plastic deformation and nonlinear damage accumulation

Elastic and plastic constitutive model. Full stress-strain model is usually used to describe the process of monotonic loading. For the process of plastic and elastic deformation which contains many loading and unloading, the incremental stress-strain model is needed.

Firstly, in the process of elastic deformation, the Hook theory is still available. Here to convert it to an incremental format[4]:

$$
d \sigma=E d \varepsilon \quad\left(\sigma<\sigma_{0}\right)
$$


When the load keeps increasing after the yield point is reached, the material will come into the stage of plastic deformation. Usually in different direction there are different yield stress and they make up a closed surface in the two-dimensional coordinate system, which is called yield surface and yield function. In the process of loading and unloading, the yield surface will move with the load history. In real process of cycled load the isotropic and kinematic hardening are existed in the same time, which is called mixed hardening. It can be expressed as:

$$
f(\sigma, \alpha, k)=[\sigma-(1-M) \alpha]^{2}-\left[(1-M) \sigma_{0}+M k\right]^{2}
$$

Where $M$ is a parameter represents the mixed degree and $M$ is between 0 and 1 .

The increment of hardening parameter in every cycle can be expressed as:

$$
d \kappa=h d \lambda
$$

Where $\mathrm{h}$ is a scalar quantity function.

When the stress in located in the boundary between elastic area and plastic area, the elastic and plastic relationship should be met in the same time, which can be expressed as:

$$
\frac{\partial f}{\partial \sigma} d \sigma+\frac{\partial f}{\partial \kappa} d \kappa=0
$$

In the process of plastic deformation, to imitate the flow rule in the hydromechanics, the relationship between stress and strain is expressed as[5]:

$$
d \varepsilon^{p}=d \lambda \frac{\partial f}{\partial \sigma}
$$

Where $f$ is the yield function and in this paper the mixed hardening is adopted.

After the definition of yield stress, hardening and flow rules, the constitutive model in the process of plastic deformation will be derivated. In the process of plastic deformation, the deformation usually consists of elastic deformation and plastic deformation:

$$
d \varepsilon=d \varepsilon^{e}+d \varepsilon^{p}
$$

In the real load process, the plastic deformation remains after the load is removed. As a result the plastic deformation increments $\mathrm{d} \varepsilon$ can be omitted here:

$$
d \sigma=E d \varepsilon^{e}
$$

Make a calculation among the function above:

$$
d \varepsilon=\frac{d \sigma}{E}+d \varepsilon^{p}=\frac{1}{E_{t}} d \sigma
$$

Where $E_{\mathrm{t}}$ is equivalent Young's modulus which is expressed as:

$$
\frac{1}{E_{t}}=\frac{1}{E} \frac{\left(\frac{\partial f}{\partial \sigma}\right)^{2} E-\frac{\partial f}{\partial K} h}{-\frac{\partial f}{\partial k} h}
$$

So far an incremental stress-strain model which expresses the deformation under the recycled load is founded.

Fatigue damage model. The general approach to deal with damage in FEA is to define a parameter $\omega$ to characterize material degradation[6]. In the research on the accumulation of damage, a nonlinear damage model is used to definite the material degradation so that the history of stress can be taken into consideration. In this paper, the Chaboche damage model is used which can be expressed by:

$$
\frac{\delta \omega}{\delta N}=\left[1-(1-\omega)^{1+\beta}\right]^{\alpha}\left[\frac{A_{I I}}{M(1-\omega)}\right]^{\beta}
$$

Where:

$$
M=M_{o}\left(1-3 b_{s} \overline{\sigma_{H}}\right), \alpha=1-a\left(\frac{A_{I I}-A_{I I}^{*}}{\sigma_{u}-\sigma_{e q_{\max }}}\right)
$$


Where $\beta, M_{0}$ and $a$ are parameters related with materials, $\sigma_{\text {eqmax }}$ is max Von Mises stress.

According to the definition of $\alpha$, the damage will occur only when $A_{I I}-A_{I I}^{*}>0$, where $A_{I I}$ and $A_{I I}^{*}$ can be expressed as:

$$
\begin{gathered}
A_{I I}=\frac{1}{2} \sqrt{\frac{3}{2}\left(S_{\max }-S_{\min }\right):\left(S_{\max }-S_{\min }\right)} \\
A_{I I}^{*}=(1-\omega) \sigma_{f}\left(1-3 b_{s} \overline{\sigma_{H}}\right)
\end{gathered}
$$

Where $S_{\max }$ and $S_{\min }$ are the deviatoric tensors of maximum and minimum stresses in the cyclic loading.

Under the uniaxial stress there is a simplification that $\mathrm{A}_{\mathrm{II}}=\Delta \sigma . \sigma_{\mathrm{f}}$ is the fatigue limit, $\sigma_{\mathrm{u}}$ is the ultimate strength, $\sigma_{\mathrm{H}}$ is the mean value of the hydrostatic stress and $b_{\mathrm{s}}$ is a parameter related with materials.

By setting damage parameter $\omega$ from 0 to 1, the fatigue life can be obtained:

$$
N_{f}=\frac{1}{(1+\beta)(1-\alpha)}\left(\frac{A_{I I}}{M}\right)^{\beta}
$$

In every stress cycle there is a damage value $\Delta \omega$. This process can be expressed as:

$$
\omega_{N+\Delta N}=\omega_{N}+\Delta \omega
$$

Coupling model. The damage parameter $\omega$ quoted before can be expressed that: In an optional unit of materials, if the area in one direction is $A$, as a result of damage the real area can be seen as $A^{*}$. So that the damage parameter $\omega$ can be expressed as:

$$
\omega=\frac{A-A^{*}}{A}
$$

Obviously when $\omega$ is set as 0 the material remains undamaged and when $\omega=1$ the material is totally ruined.

Because of the $\omega$, valid area of material when there is some load will decrease so that the real valid stress will increase. The valid stress can be expressed as:

$$
\sigma^{*}=\frac{\sigma}{1-\omega}
$$

By replacing the real stress with the valid stress so that:

$$
d \sigma^{*}=\frac{d \sigma}{1-\omega}=E_{t} d \varepsilon
$$

So far the coupling model of deformation and damage has been founded.

\section{Model development}

Under microscopic observation common metallic materials tends to show a significant distribution of grains and grain boundaries. The shape of grain is irregular and the scale of strain is not uniform. Considering the geometric properties of the process of liquid metal cooling, nucleation and crystallization, the Voronoi picture can be used to describe the microstructure of the metallic materials. As is shown in Fig.1, the metallic microstructure founded by Voronoi is very similar in shape and dimensions to the real microstructure of common metallic materials. 

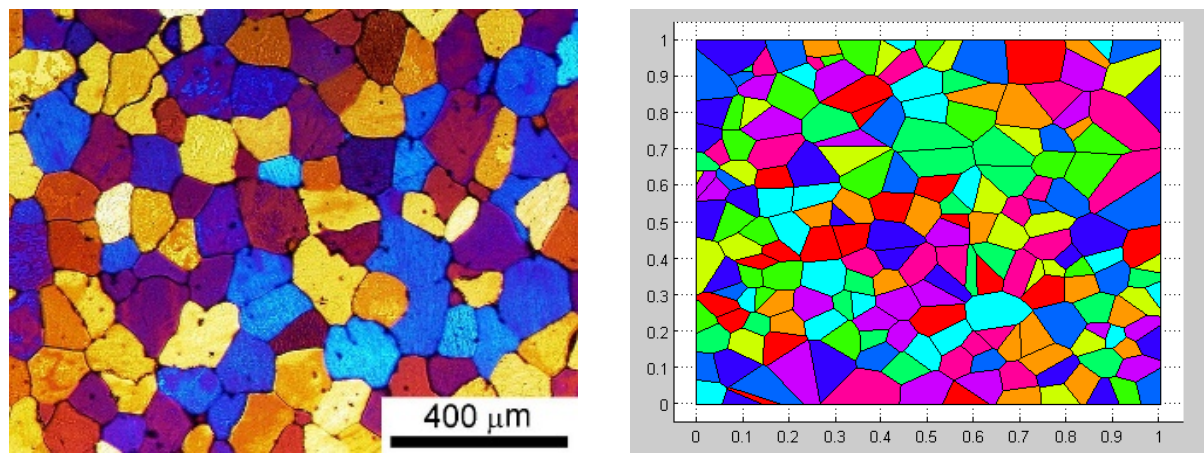

Fig. 1 Metallic microstructure and 2-D Voronoi picture

In this paper the method of mixed programming between Matlab and Python is used. In the matlab environment Voronoi picture is founded and its geometric information is output. In the Python environment built in Abaqus compiler the geometric information is received from the previous step and its reconstruction is finished. Implementation process is as follows:

To establish a two-dimensional and three-dimensional Voronoi and output its geometric information in the matlab environment. In the matlab environment MPT toolbox is called to generate two-dimensional and three-dimensional Voronoi pictures. Location and topology information of points and segments for 2-D Voronoi can be output directly.

To read and reconstruct the geometric information of materials in Abaqus environment. There is a built-in PDE interface in Abaqus which can be used to run the Python language to control the process of simulation by rpy file, including the process of modeling, loading, and other post-processing analysis step. There is also an XLRD module built in the Python language which can read the geometric information on the previous step in the format of Excel and rebuild it in the Abaqus environment so that the material microstructure characteristics required can be built in Abaqus environment.

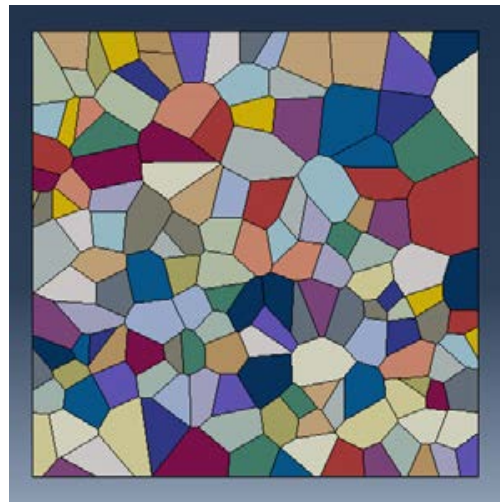

Fig. 2 2-D diagram of the new metallic microstructure

Comparing the microscopic picture of common metallic materials and new metallic microstructure established in this paper, it is concluded that these two parts share a high degree of similarity. In order to verify the accuracy of the model, the finite element model of the new microstructure is used to simulate the material properties and the results of simulation will be compared with the experimental results.

To perform a stochastic distribution of material properties in the new model. In the microstructure model, each small part can be seen approximately as an independent grain correspond to the real grain in common metallic materials. According to the approach of stochastic distribution, each grain in the material has a different mechanical property which performs the phenomenon that the yield point of each grain is different from each other. By nano-indentation experiments the theory of distribution is obtained and in the next step this theory will be introduced into the microstructure.

This part is fulfilled by Python program in the FEA software Abaqus. Firstly a series of random material properties is obtained by some module. Secondly a series of unit number is obtained by another module and the unit number is coordinated with the material property. Lastly the property will be matched with the unit one by one until all grains has been assembled with a property value. 


\section{Simulation and Experiments}

After the simulation model is founded, some FEA analysis is performed on the tensile and cycled experiment. To verify the simulation some experiment is also performed and the tensile and cycled curve is obtained from the experiment to make a contrast between simulation and experiment.

Simulation. To reduce the amount of work and to simplify the calculation process, two-dimensional microstructure is used to conduct the simulation of tensile test. In the simulation process, in accordance with the periodic boundary conditions, some areas of sample are picked up with load on the sample upper surface. The simulation model is shown and some parameters of the material part are set as follows:

Table 1 Settings of scale parameters for materials in simulation

\begin{tabular}{cc}
\hline Parameters & Values \\
\hline Sample size & $1 \mathrm{~mm} * 1 \mathrm{~mm}$ \\
Number of grains & 400 \\
Average grain scale & $50 \mathrm{um}$ \\
\hline
\end{tabular}

Take the steel 40Cr material as an example, to set the parameters of the mechanical properties of the material in the material module of Abaqus as follows:

Table 2 The relevant material properties in preprocessing

\begin{tabular}{cc}
\hline Paramaters & Values \\
\hline Young's modulus & $210 \mathrm{GPa}$ \\
Poisson's ratio & 0.33 \\
Yield limit & $780 \mathrm{MPa}$ \\
Tensile strength & $950 \mathrm{MPa}$ \\
\hline
\end{tabular}

After the definition of materials, modeling, loading, meshing and other pre-processing step, the peak load of $750 \mathrm{MPa}$ is set. Simulation of tensile test process is performed. After the simulation process the state of stress in the whole and partial specimen is shown as Fig. 3. The tensile curve in the state of simulation after the processing of data from the tensile simulation is as follows. As is seen from the graph that each grain has a different stress state. Compared with the traditional modeling methods, the new model can reflect the microscopic structure of the material better under load force and deformation.

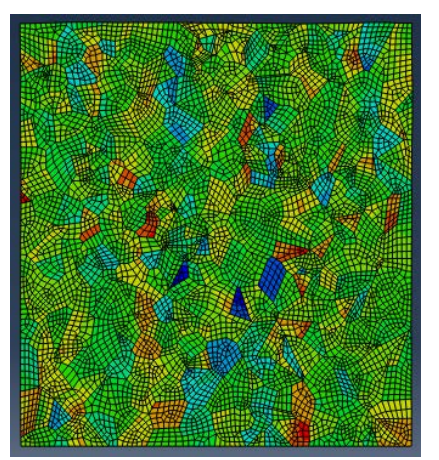

Fig. 3 Tensile test results of the finite element simulation

After the simulation of tensile test, the simulation of tension and compression testing under cycled load is performed. As is shown in Fig. 4, the whole process consists of three stages: Firstly, in the beginning stage of the load, the plastic deformation and damage accumulation begin to increase. At this time the deformation is not apparent. Secondly, during the load, the plastic deformation and damage accumulation continue to increase and the deformation is increasing according to the curve. Lastly, in the end of the load, the deformation and damage tends to become very large and the material is due to crack. 

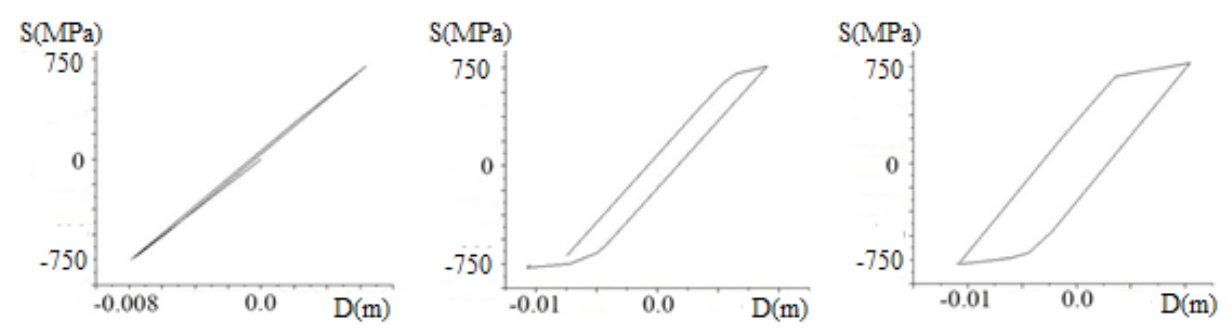

Fig. 4 Tensile and compress results of the FEA

Experiment. In order to verify the accuracy of the simulation results, the steel $40 \mathrm{Cr}$ is taken as test material to perform a series of tensile experiment. And some specimen for tensile experiment is designed and manufactured in accordance with the national standard and some tensile test is performed with MTS810 hydraulic fatigue testing machine. Picture of these specimens is shown as follows. After the tensile experiment, comparing the tensile curve obtained from the simulation results and experimental results, the accuracy of the new microstructure model can be verified.

Comparing the tensile curve obtained from the simulation results and the test results, it can be found that the rising and changing trend between the two curves is consistent fundamentally. The conclusion can be made that description of metallic materials microstructure with the new micro-structure model is credible and accurate.

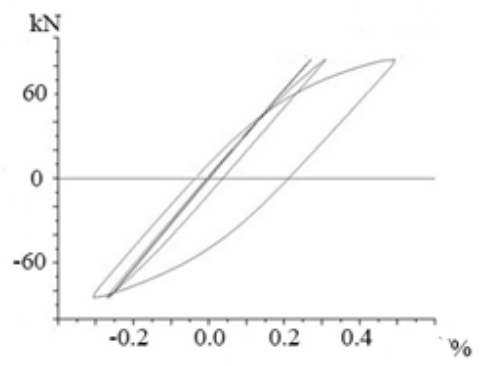

Fig. 5 Hysteresis curves under 750MPa cyclic loading

As is shown in the figure, under the plastic hardening and repeat of the stress, the strain under symmetric cyclic loading is increasing. When the load turns to asymmetrical load, there is almost no plastic deformation and only some deformation caused by the ratcheting effects. The results obtained from the experiments show that the simulation model can substitute the real specimen to some degree and the simulation results is similar to the experimental results.

\section{Conclusion}

With the introduction of Voronoi picture in the modeling process to describe the material microstructure and stochastic distribution of material properties, the new microstructural finite element model is able to restore the microstructure of the material better. The tensile test and compressing test results show that the appropriate use of new microstructure model in finite element simulation can get accurate simulation results. In some occasions where more accurately restored material microstructure is needed, the new micro-structural finite element model established in this paper can get more accurate results.

\section{Acknowledgement}

This material is based upon work supported by the National Natural Science Foundation of China (51405101), the research and innovation fund of Harbin Institute of Technology (Grant Number: HIT.NSRIF.2015 053), the China Postdoctoral Science Foundation (Grant Number: 2014M561340 and 2016T90277) and Heilongjiang Postdoctoral Fund (Grant Number: LBH-Z14100).

\section{References}

[1] S. Keshavarz, S. Ghosh. Multi-scale crystal plasticity finite element model approach to modeling nickel-based superalloys. Acta Materialia. 2013: (61): 6549-6591. 
[2] M. Naderi, S.H. Hoseini, M.M. Khonsari. Probabilistic simulation of fatigue damage and life scatter of metallic components, International Journal of Plasticity. 2013: (43): 101-115.

[3] G. Deodatis. Stochastic FEM sensitivity analysis of nonlinear dynamic problemsOriginal Research Article. Probabilistic Engineering Mechanics. 1989:4 (3): 135-141.

[4] S.A. Ramu, R. Ganesan. Response and stability of a stochastic beam-column using stochastic FEM. Computers \& Structures. 1995:54 (2):207-221.

[5] G.Z. Kang, Q. Gao. Uniaxial and non-proportionally multiaxial ratcheting of U71Mn rail steel: experiments and simulations. Mechanics of Materials, 2002:34 (12): 809-820.

[6] B.K. Chun, H.Y. Kim, J.K. Lee. Modeling the Bauschinger effect for sheet metals, part II: applications. International Journal of Plasticity. 2002:5 (18):571-595. 